Pure \& Appl. Chem., Vol. 57, No. 5, pp. 701-708, 1985.

Printed in Great Britain.

(C) 1985 IUPAC

\title{
Stable isotopes in carotenoid biochemistry
}

\author{
George Britton \\ Department of Biochemistry, University of Liverpool, P.O. Box 147, Liverpool, \\ L69 3BX, U.K.
}

\begin{abstract}
The use of stable isotopes in studies of carotenoid biosynthesis is reviewed, beginning with early work with 180 which defined the origin of oxygen in a range of xanthophylls. A series of experiments from our own laboratory is then described, in which ${ }^{2} \mathrm{H}$ and ${ }^{13} \mathrm{C}$ have been used to investigate the stereochemistry of cyclization to form $\mathrm{C}_{40}$ and $\mathrm{C}_{50} \boldsymbol{\beta}$ - and $\boldsymbol{\varepsilon}$-ring carotenoids. Novel procedures involving $2 \mathrm{H}$ labelling from $2 \mathrm{H}_{2} \mathrm{O}$ have been used to demonstrate carotenoid transformations during biosynthesis and chloroplast development and to define the stereochemistry of the $\mathrm{H}^{+}$attack at C-2 which initiates carotenoid cyclization. Procedures by which microorganisms are allowed to metabolize $\left[{ }^{1} \mathrm{H}\right]$-glucose, in ${ }^{2} \mathrm{H}_{2} \mathrm{O}$, or $\left[{ }^{13} \mathrm{C}_{6}\right]-$ glucose to make endogenous specifically labelled substrates are described. These methods allow the carotenoid C-I methyl substituents to be distinguished even when incorporation of exogenous labelled substrates is not possible, and have been used to show that the stereochemistry of cyclization to form the $\boldsymbol{\beta}$ - and $\boldsymbol{\varepsilon}$-rings of the $\mathrm{C}_{40}$ carotenoids zeaxanthin and lutein is the same.
\end{abstract}

\section{INTRODUCTION}

In the field of retinaldehyde-proteins, ${ }^{13} \mathrm{C}$ and $2 \mathrm{H}$ labelled analogues have been prepared and used in NMR, IR and Raman spectroscopic studies of the molecular interactions and mechanisms of the structural changes that occur during the visual pigment and bacteriorhodopsin cycles (Ref. 1). No such studies on carotenoid-proteins have been reported even though similar structural features and interactions may be crucial to the mechanism of the spectral shift in animal astaxanthin-proteins, and possibly also to the functioning of carotenoids in other pigment-protein complexes, e.g. in the photosynthetic apparatus of plants and bacteria. In carotenoid biochemistry, the use of stable, heavy isotopes has been restricted to the study of biosynthetic pathways and the mechanism of biosynthetic reactions, and even then they have been much less widely used than the radioisotopes ${ }^{14} \mathrm{C}$ and ${ }^{3} \mathrm{H}$.

\section{EARLY WORK WITH 180}

Incorporation of ${ }^{18} \mathrm{O}$ from ${ }^{18} \mathrm{O}_{2}$ and $\mathrm{H}_{2}{ }_{2}^{18} \mathrm{O}$ into xanthophylls

Inevitably, the first stable isotope work in the carotenoid field was with ${ }^{18} \mathrm{O}$, there being no suitable radioactive isotope of this element. The questions asked concerned the origin of the oxygen functions present in a range of xanthophylls, specifically whether these arose from molecular $\mathrm{O}_{2}$ or from water. With the photosynthetic bacterium Rhodopseudomonas sphaeroides, it is well known that the main pigment in anaerobic cultures is spheroidene which, in the presence of air, is converted into the 2-oxo derivative spheroidenone. Shneour (Ref.2) demonstrated clearly that the oxygen atom of the oxo group came from molecular ${ }^{18} \mathrm{O}_{2}$. A series of experiments to determine the origin of the hydroxy and epoxy oxygens of the leaf xanthophylls, lutein, violaxanthin, neoxanthin and antheraxanthin, gave less conclusive results (Refs. 3-7) In this work, with leaves, photosynthetic particles derived from them, and the green alga Chlorella, ${ }_{18} \mathrm{O}_{2}$ and $\mathrm{H}_{2}{ }^{18}$ O were used with comparatively low enrichment (u5\%), and 180:160 ratios in the xanthophylls were determined by $\mathrm{MS}$ assay of the $\mathrm{CO}_{2}$ obtained by pyrolysis. With Chlorella, some $18 \mathrm{O}$ enrichment from $18 \mathrm{O}_{2}$ was obtained for lutein, violaxanthin and neoxanthin, and slight enrichment in neoxanthin was seen when $\mathrm{H}_{2}{ }^{18} \mathrm{O}$ was used. The conclusion was drawn that the hydroxy groups arose from molecular $\mathrm{O}_{2}$ whereas the epoxy groups originated from water. Conflicting results were obtained concerning the stimulation by light of the incorporation of $18 \mathrm{O}$ from $\mathrm{H}_{2} 18 \mathrm{O}$ into violaxanthin. Later work (Ref. 7) showed incorporation of $18 \mathrm{O}_{2}$ into antheraxanthin (assumed into the epoxy group) in leaves in the dark. No distinction was made at that time between incorporation of oxygen during biosynthesis or during operation of the violaxanthin cycle. Incorporation of $18 \mathrm{O}$ was also obtained into the carboxylic acid torularhodin from the yeast Rhodotorula rubra grown in the presence of $5-6 \%$ atom excess of $18 \mathrm{O}_{2}$. From the small enrichment obtained, it was concluded that one $18 \mathrm{O}$ atom was incorporated into the carboxy group (Ref. 8). Now that highly enriched ${ }^{18} \mathrm{O}_{2}$ and $\mathrm{H}_{2}{ }^{18} \mathrm{O}$ are more readily available and instrumentation for mass spectrometric assay is so greatly improved, these problems should be reinvestigated, and the carotenoid molecules themselves assayed in order to prove 
the incorporation and location of the 180 atoms. It is unfortunate that the practical problems associated with 17O NMR spectroscopy appear to preclude the useful application of this isotope for this work (Ref. 9).

\section{WORK WITH ${ }^{2}$ H AND ${ }^{13} \mathrm{C}$}

\section{Synthetic $\left[2 \mathrm{H}_{3}\right]$-lycopene and carotenes}

As in other branches of biochemistry, it is the stable heavy isotopes of carbon and hydrogen that are now proving most useful in studies of carotenoid biosynthesis. First at Madison and later at Liverpool (Refs. 10, II) Prof. Eugster described the synthesis and properties of the stereospecifically labelled $\left[16,16{ }^{\prime}-2 H_{6}\right]-\beta, \beta-, \quad-\beta, \varepsilon-$, and $-\gamma, \gamma$-carotenes. The $l_{H}$ NMR properties of these chiral cyclic carotenes were determined, making it possible for the chirality of samples biosynthesized from the $\left[2 \mathrm{H}_{6}\right]$-lycopene to be elucidated. Unfortunately, work with carotenogenic enzyme systems has not yet advanced to the stage when conversion of this lycopene substrate into cyclic carotenes can be achieved efficiently enough to give sufficient product for NMR assay and hence permit determination of the stereochemical behaviour of the C-l methyl groups during cyclization. This problem has, however, been addressed recently by a conventional ${ }^{13} \mathrm{C}$ labelling procedure (Ref. 12).

Incorporation of [2-13 C]-mevalonate into lycopene, zeaxanthin and c.p. 450

The high-carotenoid Flavobacterium strains RI519 and R1560 will incorporate mevalonic acid (MVA) efficiently into their main pigment, zeaxanthin or, when grown in the presence of the cyclization inhibitor nicotine, into the acyclic precursor lycopene. Flavobacterium cultures were therefore grown, with and without nicotine, in the presence of [2-13C]-MVA. The lycopene and zeaxanthin, repectively, were isolated and their $13 \mathrm{C}-\mathrm{NMR}$ spectra determined. The spectrum of lycopene showed $13 \mathrm{C}$ enrichment at C-4, C-8 and C-12 (40.8, 135.5 and 137.5 p.p.m.), and at C-16 (25.7 p.p.m), i.e. the C-1 methyl substituent trans to the main carbon chain of the molecule. This result confirmed the expected isoprenoid labelling pattern previously established after painstaking degradation of $14 \mathrm{C}$-labelled carotenoids (Ref. 13), and in particular it proved that the methyl substituents at C-I of lycopene retain their individuality during the biosynthesis. From the ${ }^{13} \mathrm{C}$ NMR spectrum of the enriched zeaxanthin it could again be seen that the ${ }^{13} \mathrm{C}$ enrichment was at $\mathrm{C}-4, \mathrm{C}-8, \mathrm{C}-12$ and one of the $\mathrm{C}-1$ methyl substituents. Detailed NMR work (Dr. G. Englert) has proved that the enhanced signal at 28.81 p.p.m. is that due to $\mathrm{C}-16$, i.e. the axial, $1 \alpha$, methyl substituent, the equatorial, $1 \beta$ methyl group, (C-17, 30.34 p.p.m.) showing no enrichment. The stereochemical behaviour of the C-I methyl groups during cyclization to form zeaxanthin was therefore established. A similar experiment in which Corynebacterium poinsettiae was incubated with [2-13C]-MVA gave a ${ }^{13} \mathrm{C}$-enriched product, the $C_{50}$ diol 'c.p.450. Enhancement was again seen in the higher field C-1 methyl signal (22.54 p.p.m.), tentatively assigned to the axial $1 \alpha$ substituent, $C-16$. The signal at 28.38 p.p.m. assigned to the $\beta$-substituent $C-$ 17 , showed no enhancement. If the assignment is correct the final disposition of the C-I methyl substituents is the same in the $C_{40}$ and $C_{50} \beta$-ring carotenoids.

Use of $2 \mathrm{H}$ labelling from ${ }^{2} \mathrm{H}_{2} \mathrm{O}$

Demonstration of biosynthetic pathways. It is believed that the cyclization reaction is initiated by electrophilic attack by a proton ( $C_{40}$ series) or a $C_{5}$ unit ( $C_{50}$ series) at $C-2$ of the acyclic precursor. Therefore, in order to define fully the stereochemistry of the initial stage of cyclization, the orientation of the incoming group at C-2 must be established. In the $C_{50}$ series, CD correlation and NMR analysis have established (Refs. 14-16) that the $C_{5}$ substituent at $C-2$ occupies the equatorial $\alpha$-position, so that the $\beta, \beta$-diol, c.p.450, the $\varepsilon, \varepsilon$-diol decaprenoxanthin, and the $\gamma, \gamma$-diol sarcinaxanthin all have the configuration (2R, 2' $)$ ). The stereochemistry of $\mathrm{H}^{+}$attack at $\mathrm{C}-2$ during cyclization to form the $\beta$-and $\varepsilon$-rings in the $\mathrm{C}_{40}$ series has now been established by a procedure involving deuterium labelling from $2 \mathrm{H}_{2} \mathrm{O}$. This procedure has also been very useful in giving direct proof of some carotenoid transformations during biosynthesis and during chloroplast development.

The basis of these experiments lies in the discovery some years ago that several microorganisms could be adapted to grow in medium in which the water was replaced virtually completely with heavy water, deuterium oxide, ${ }^{2} \mathrm{H}_{2} \mathrm{O}$. Crespi, Katz and coworkers had maintained and grown the green algae Chlorella and Scenedesmus under these conditions for long periods (Ref. 17), and isolated fully deuteriated $\alpha$ - and $\beta$-carotenes for spectroscopic study (Ref. 18). We have adapted this work, the simple concept assumed being that, if an organism can be cultured de novo in $2 \mathrm{H}_{2} \mathrm{O}$, then cultures will be able to survive and continue to grow and develop when transferred from ordinary medium $\left(\mathrm{l}_{2} \mathrm{O}\right)$ to $2 \mathrm{H}_{2} \mathrm{O}$. The experimental protocol is thus simple; the organism is first cultured in normal medium $\left(\mathrm{H}_{2} \mathrm{O}\right)$ under one set of conditions and then transferred to $2 \mathrm{H}_{2} \mathrm{O}$ medium when the growth conditions are altered so that changes occur in metabolism or development. A good illustration is provided by an experiment to demonstrate the direct conversion of lycopene into zeaxanthin in vivo in the Flavobacterium strain RI519 (Ref. 19). The experimental procedure consisted of first growing the Flavobacterium in ordinary $\left(\mathrm{l}_{\left.\mathrm{H}_{2} \mathrm{O}\right)}\right.$ medium in the presence of the cyclization inhibitor nicotine so that the acyclic precursor lycopene accumulated, then washing out the nicotine and transferring the cells to $2 \mathrm{H}_{2} \mathrm{O}$. The zeaxanthin thus produced was isolated and assayed by mass spectrometry. Three kinds of zeaxanthin molecule are

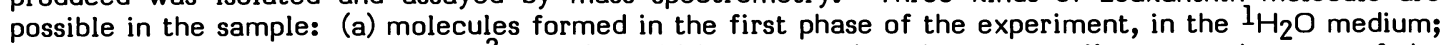
these would obviously contain no $2 \mathrm{H}$, and would be present in only very small amounts because of the inhibitor, (b) molecules formed de novo in the second phase of the experiment; these would contain

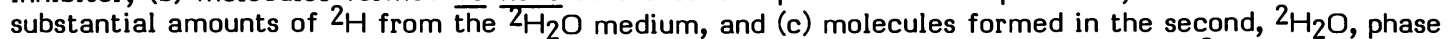
from $\left[{ }^{l} \mathrm{H}\right]$-lycopene accumulated in the first phase; this zeaxanthin would contain two ${ }^{2} \mathrm{H}$ atoms, at $\mathrm{C}-2$ and $\mathrm{C}^{\prime}$, introduced during cyclization initiated by $2 \mathrm{H}^{+}$from the medium. The different zeaxanthin species can easily be identified in the mass spectrum and the relative amounts determined. The 
experiment showed that substantial new synthesis of zeaxanthin had taken place in the $2 \mathrm{H}_{2} \mathrm{O}$ medium, resulting in molecules containing an average of 12-16 $2 \mathrm{H}$ atoms. The main species present, however, especially if the second phase incubation was for not more than 12 hours, was $\left[{ }^{2} \mathrm{H}_{2}\right]$-zeaxanthin. This

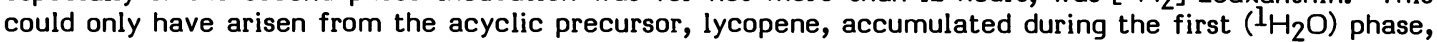
a result which therefore proved directly the biosynthetic conversion of lycopene into zeaxanthin.

Similar experiments have since been performed with the photosynthetic bacteria Rhodopseudomonas sphaeroides and Rhodomicrobium vannielii (Ref. 20). These organisms make acyclic xanthophylls, notably spheroidene and hydroxyspheroidene in Rps. sphaeroides and rhodopin in Rm. vannielii. The introduction of the $\mathrm{C}-\mathrm{l}$ hydroxy groups, like cyclization, is inhibited by nicotine, and precursors accumulate, neurosporene in Rps. sphaeroides and lycopene in $\mathrm{Rm}$. vannielii. When inhibited cultures were washed free from the inhibitor and reincubated in ${ }^{2} \mathrm{H}_{2} \mathrm{O}$ medium, the spheroidene and hydroxyspheroidene samples that were then isolated contained substantial amounts of ${ }_{2} \mathrm{H}_{1}$ species with the $2 \mathrm{H}$ atom located at $\mathrm{C}-2$. As before, this provided direct proof of the biosynthetic conversions of neurosporene into spheroidene and of lycopene into rhodopin, thus confirming the operation of the postulated biosynthetic pathways.

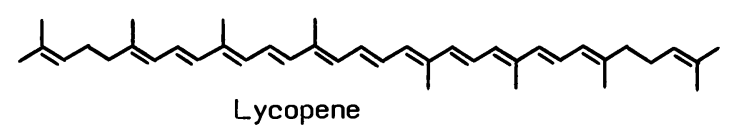

Lutein

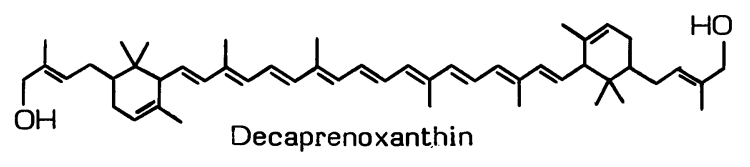

Decaprenoxanthin

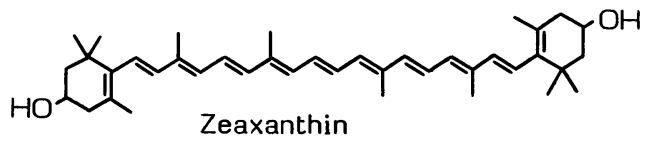

Zeaxanthin

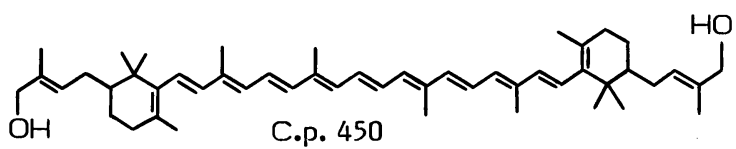

C.p. 450<smiles>CC(O)(CCO)CC(=O)[O-]</smiles>

Mevalonate (MVA)

Stereochemistry of introduction of $\mathrm{H}$ at C-2 during cyclization. In the formation of $\left[2 \mathrm{H}_{2}\right]-z e a x a n t h i n$ in Flavobacterium by cyclization in $2 \mathrm{H}_{2} \mathrm{O}$ of accumulated lycopene, the introduction of $2 \mathrm{H}$ at $\mathrm{C}-2$ in each ring formed is expected to be stereospecific and to produce a chiral centre at C-2. The C-2 chirality (S) of the $\left[{ }^{2} \mathrm{H}_{2}\right]$-zeaxanthin has been determined by $\mathrm{I}_{H}$ NMR spectroscopy and the stereochemistry of introduction of $2 \mathrm{H}$ at $\mathrm{C}-2$ thus established (Ref. $2 \mathrm{l}$ ). The $\mathrm{l}_{\mathrm{H}} \mathrm{NMR}$ spectrum of the $\left[2,2^{\prime}-{ }^{2} \mathrm{H}_{2}\right]$-zeaxanthin from Flavobacterium was very similar to that of the normal unlabelled compound except that the signal attributed to the axial $(\alpha)$ hydrogen at C-2 (1.48 p.p.m.) was missing, whereas the C-2 equatorial proton signal at 1.77 p.p.m. was still present, though modified to a broad singlet due to removal of the normal coupling with the 2-axial proton. This therefore established the stereochemistry of the first step, the $\mathrm{H}^{+}$attack, in the formation of the $\beta$-ring in the $\mathrm{C}_{40}$ carotenoids and, when taken with the result of the incorporation of [2-13 C]-MVA (above), it defines fully the stereochemistry of the cyclization reaction in the biosynthesis of zeaxanthin in Flavobacterium.

The stereochemistry of $\mathrm{H}^{+}$attack at $\mathrm{C}-2$ during cyclization to form the $\varepsilon$-ring in lutein has also been established by employing a somewhat similar strategy with a mutant strain, $\mathrm{PGl}$, of the green alga Scenedesmus obliquus. When grown in the light this strain is indistinguishable from the wild type, having normal chloroplasts and a normal complement of cyclic carotenoids, $\alpha$ - and $\beta$-carotenes, lutein, violaxanthin, neoxanthin and loroxanthin. When grown in the dark, however, PGI is unable to make normal chloroplasts, has little or no chlorophyll and accumulates acyclic precursors, phytoene and especially $\zeta$-carotene, in place of the normal cyclic carotenoids. On illumination of the dark-grown cultures, the development of chloroplasts is completed and cyclic carotenoids are formed, apparently at the expense of the accumulated acyclic precursors (Ref. 22). This is therefore another system in which carotenoid synthesis, in this case as part of chloroplast development, can be made to take place in two stages. The experimental procedure is very simple; the algae are grown in the dark in ordinary $\left(\mathrm{l}_{\mathrm{H}_{2}} \mathrm{O}\right)$ medium, then transferred to $2 \mathrm{H}_{2} \mathrm{O}$ and illuminated. Any cyclic carotenoids formed in the light from the acyclic precursors that accumulated in the dark will be $\left[{ }^{2} \mathrm{H}_{2}\right]$-species (one ${ }_{2} \mathrm{H}$ atom introduced for each cyclization) whereas cyclic carotenoids synthesized in the light de novo, i.e. from $\mathrm{CO}_{2}$ and $2 \mathrm{H}_{2} \mathrm{O}, w^{2}$ be extensively deuteriated. As with the Flavobacterium system, the two kinds of carotenoid molecules can be distinguished and assayed by mass spectrometry. Also, as in the case of Flavobacterium, the $2 \mathrm{H}$ introduction at C-2 during cyclization of the accumulated acyclic precursor will be stereospecific, and the chirality at $\mathrm{C}-2$ of the products can be determined by $l_{H}$ NMR spectroscopy. The main xanthophyll formed by S.obliquus PGI under these conditions is lutein, which thus provides an opportunity to determine the stereochemistry of formation of the $\varepsilon$-ring (Ref.23). A sample of lutein prepared from 
S.obliquus PGl under these conditions so that the $\left[2 \mathrm{H}_{2}\right]$-species predominated was analysed by $\mathrm{l}_{\mathrm{H}} \mathrm{NMR}$ spectroscopy. Of the signals attributed to the 3-hydroxy- $\beta$-ring, only that of the 2 -axial hydrogen (1.48 p.p.m.) was missing, confirming the location of the $2 \mathrm{H}$ atom at this position, in agreement with the result previously obtained for zeaxanthin in Flavobacterium. The other signal missing from the spectrum was that at 1.84 p.p.m. assigned to the $2^{\prime}$-equatorial hydrogen of the $\varepsilon$-ring. The chirality at $C-2^{\prime}$ of the $\varepsilon$ ring, was therefore $(\underline{S})$, the same as that established for $\mathrm{C}-2$ of the $\beta$-ring. That the $2 \mathrm{H}$ atom introduced occupies an axial position in the $\beta$-ring but an equatorial position in the $\varepsilon$-ring is due to the different overall conformation of the two rings, determined by the 3- and 3'-hydroxy substituents adopting a quasi- equatorial configuration. The stereochemistry of $\mathrm{H}^{+}$attack is therefore the same in the formation of the $\beta$ - and $\varepsilon$-rings in the $\mathrm{C}_{40}$ carotenoids, but opposite to that of the introduction of $\mathrm{C}_{5}$ units in the $\mathrm{C}_{50}$ series.

Carotenoid transformations during chloroplast development. The ability to distinguish cyclic carotenoid molecules synthesized de novo from those which are formed in the light from $\zeta$-carotene etc. that accumulated in the dark has provided an opportunity to use PGI to look at a number of aspects of the biosynthesis of carotenoids in relation to chloroplast development in this organism, as the dark-grown cultures are allowed to 'green' in the light. A series of experiments with this system has shown the following (Refs. 24,25):- (i) in the first stages of greening (up to 5 hours) the cyclic carotenoids isolated, both carotenes and xanthophylls, are $\left[\mathrm{H}_{2}\right]$-species formed from the accumulated $\zeta$-carotene. (ii) In the later stages of greening ( 5 hours onwards) the cyclic carotenoids formed are almost entirely the extensively deuteriated species, formed de novo from $\mathrm{CO}_{2}$ and $2 \mathrm{H}_{2} \mathrm{O}$. (iii) This synthesis de novo is prevented by the protein synthesis inhibitors chloramphenicol and cycloheximide, which affect protein synthesis inside and outside the chloroplast, respectively, showing that enzyme synthesis is required before 'new' carotenoid molecules can be produced. (iv) The formation of cyclic carotenoids from accumulated $\boldsymbol{\zeta}$-carotene is not prevented by these inhibitors. The cyclase enzymes must therefore be present in the dark-grown cultures but require light for activation. ( $v$ ) The inhibition of new synthesis by cycloheximide is removed to a considerable extent if glucose is provided as carbon source substrate. Presumably the synthesis de novo can then continue through the action of the enzymes responsible for making the carotenoid skeleton in the dark. The inhibitor blocks formation of the enzymes of $\mathrm{CO}_{2}$ fixation, thereby preventing the use of $\mathrm{CO}_{2}$ for carotene synthesis. The inhibition by chloramphenicol is not alleviated by the addition of glucose. Chlorophyll synthesis is totally blocked by both inhibitors, the addition of glucose having no effect. (vi) The time courses of formation of the $2 \mathrm{H}_{2}$ and $2 \mathrm{H}_{n}$ species are different for carotenes and xanthophylls, indicating a fundamental difference at least in the control of the synthesis of the two groups of carotenoids, if not in the synthesis itself. (vii) The 'secondary' ketocarotenoids, e.g. 3-hydroxyechinenone, that accumulate outside the chloroplast when Scenedesmus is subjected to conditions of mineral deficiency (especially of nitrogen) are also produced largely as $\mathrm{ZH}_{2}$ species. Therefore the accumulated $\boldsymbol{\zeta}$-carotene can serve as a precursor to both the chloroplast cyclic carotenoids and the extraplastidic secondary ketocarotenoids.

\section{GENERATION OF SPECIFICALLY ${ }^{2}$ H AND ${ }^{13}$ C LABELLED MEVALONATE AND ACETATE IN VIVO}

When suitable substrates, especially MVA, can be incorporated efficiently into carotenoids, the use of ${ }^{13} \mathrm{C}$ labelling as outlined above for zeaxanthin in Flavobacterium to demonstrate isoprenoid labelling patterns and the stereochemical behaviour of the $\mathrm{C}-1$ methyl substituents is straightforward, almost routine, provided the ${ }^{13} \mathrm{C}$ NMR spectrum can be assigned rigorously. Unfortunately this procedure cannot be applied universally since many organisms, especially bacteria, will not incorporate the substrate at all, or at least not efficiently enough to give the required degree of enrichment. To investigate the stereochemistry of biosynthetic reactions in these systems, other strategies need to be devised and developed. We have now successfully used two such procedures, one with ${ }^{2} \mathrm{H}$, the other with ${ }^{13} \mathrm{C}$ labelling, which essentially rely on the production and utilization of specifically labelled substrates in vivo.

\section{Growth of microorganisms on $2 \mathrm{H}_{2} \mathrm{O}$ and $\left[{ }^{l} \mathrm{H}\right]$-glucose}

Demonstration of labelling pattern in lycopene. The first of these procedures follows from the $2 \mathrm{H}_{2} \mathrm{O}$ methods described above, and is based on the observation that synthesis de novo in ${ }^{2} \mathrm{H}_{2} \mathrm{O}$ medium was frequently found not to give fully deuteriated carotenoid products (e.g. $\left[2 \mathrm{H}_{5}\right]-\beta$-carotene) but species with variable numbers of $2 \mathrm{H}$ atoms and also appreciable amounts of $\mathrm{l}_{\mathrm{H}}$. Thus Flavobacterium RI519 grown in $2 \mathrm{H}_{2} \mathrm{O}$ medium produced a range of zeaxanthin species with an average of $12 \mathrm{2} \mathrm{H}$ atoms per molecule, i.e. $\mathrm{C}_{40} \mathrm{l}_{\mathrm{H}_{44}} 2_{\mathrm{H}_{12}} \mathrm{O}_{2}$, whereas zeaxanthin from Flavobacterium $\mathrm{R} 1560$ had an average of 30 $2 \mathrm{H}$ atoms, i.e. was $\mathrm{C}_{40} \mathrm{I}_{26} \mathrm{H}_{30} \mathrm{O}_{2}$, decaprenoxanthin from Flavobacterium (Cellulomonas) dehydrogenans had an average composition $\mathrm{C}_{50} \mathrm{l}_{\mathrm{H}_{42}}{ }^{2} \mathrm{H}_{30} \mathrm{O}_{2}$, and lutein from Scenedesmus obliquus was virtually fully deuteriated $\left(\mathrm{C}_{40} \mathrm{l}_{\mathrm{H}_{1}}{ }^{2} \mathrm{H}_{46} \mathrm{O}_{2}-\mathrm{C}_{40}{ }^{2} \mathrm{H}_{56} \mathrm{O}_{2}\right)$. With light-grown Scenedesmus, the carbon source is $\mathrm{CO}_{2}$, so that essentially all the hydrogen comes from the medium and the carotenoids formed are virtually fully deuteriated. In the other cases, the medium also had ${ }^{2} \mathrm{H}_{2} \mathrm{O}$ as the only water, but the carbon source substrate on which the organism was growing, commonly glucose, was normal, i.e. contained. $l_{H}$. The differences in the relative amounts of $l_{H}$ and $2_{H}$ reflect the differences in intermediary metabolism by which the carbon source is used to form biosynthetic building units. The overall pathway can be summarized as 
the important feature being that acetate is a significant intermediate. It is obvious that, when acetate is formed from glucose, $l_{H}$ from this glucose can only be retained in the $\mathrm{CH}_{3}$ group (C-2), and the extent to which it is retained will depend upon the route by which the substrate is metabolized to acetate. Therefore in a molecule derived from this acetate any $l_{H}$ could be present only at those positions which originated as the acetate $\mathrm{CH}_{3}$ group; any hydrogen at positions originating from the $\mathrm{COO}^{-}$group of acetate would necessarily be introduced later, from the medium, as ${ }_{2} \mathrm{H}$. In MVA, retention of $\mathrm{l}_{\mathrm{H}}$ would therefore be expected at $\mathrm{C}-2, \mathrm{C}-4$ and the $\mathrm{C}-3^{\prime}$ methyl group, with only $2 \mathrm{H}$, from the medium, at $\mathrm{C}-5$. The predicted labelling pattern in lycopene formed from this has been confirmed by the $l_{H} N M R$ spectrum of a sample isolated from Flavobacterium $\mathrm{RIS19}$ grown on $2 \mathrm{H}_{2} \mathrm{O}$ and $\left[\mathrm{l}_{\mathrm{H}}\right]$-glucose, in the presence of nicotine. The NMR signals at $6.49,6.63$ and 6.64 p.p.m., assigned to the protons at C-7, II and 15 (from $\mathrm{C}-5$ of MVA) were absent due to virtually complete replacement by ${ }^{2} \mathrm{H}$. The presence of $2 \mathrm{H}$ in place of $l_{H}$ at these positions also reduces the possibilities of $l_{H}-l_{H}$ coupling, so that the form of other signals in the spectrum was changed, e.g. signals that were normally doublets due to coupling with a proton derived from C-5 of MVA became singlets.

The main aim of the experiment, however, was to attempt to distinguish between the two methyl substituents at $\mathrm{C}-\mathrm{I}$ of the carotenoid. The key to this lies in the isomerization of isopentenyl pyrophosphate (IPP) and dimethylallyl pyrophosphate (DMAPP) in the early stages of the pathway after MVA. The isomerization of IPP to DMAPP will result in the uptake of a $2 \mathrm{H}$ atom into the methyl group produced (formerly C-2 of MVA) but not into the other methyl group (formerly C3' of MVA) which is not involved in the isomerization. An equilibrium is maintained between IPP and DMAPP and the reversible isomerization may occur repeatedly before these compounds are used by the prenyl transferase enzymes to construct the tetraterpenoid skeleton of the carotenoid. Each time an isomerization occurs, more $2 \mathrm{H}$ from the medium will replace $1_{H}$ at $C-2$ (equivalent to $C-4$ of MVA), when DMAPP $\rightarrow$ IPP and at $C-4$ (equivalent to C-2 of MVA), when IPP $\longrightarrow$ DMAPP. The C-3' methyl group is not involved in the isomerization and will therefore retain its $1_{\mathrm{H}}$. Essentially then, this procedure relies on the production and utilization in vivo of MVA that can be considered to be labelled at C-5 (fully deuteriated) and /or at C-3' (greatest retention of $l_{H}$ ). The retention of $l_{H}$ in any isoprenoid compound should therefore be greater at positions derived from C-3' of MVA than at any other positions in the molecule, In particular it should be possible to distinguish between the C-I methyl substituents of a carotenoid, one of which will arise from C-3' of MVA, the other from C-2. This was clearly shown in the case of this lycopene (Fig IA); the $l_{H}$ NMR signal at 1.69 p.p.m. from the C-16 methyl group (the C-1 methyl substituent trans to the main carbon chain and derived from $C-2$ of MVA) was very weak due to extensive $2 \mathrm{H}$ replacement, whereas that at 1.62 p.p.m. due to the C-17 methyl group (cis to the main carbon chain and derived from C-3' of MVA) remained of high intensity, as did the signals at 1.82 and 1.97 p.p.m. due to the C-18, 19 and 20 methyl groups which are also derived from C-3' of MVA.
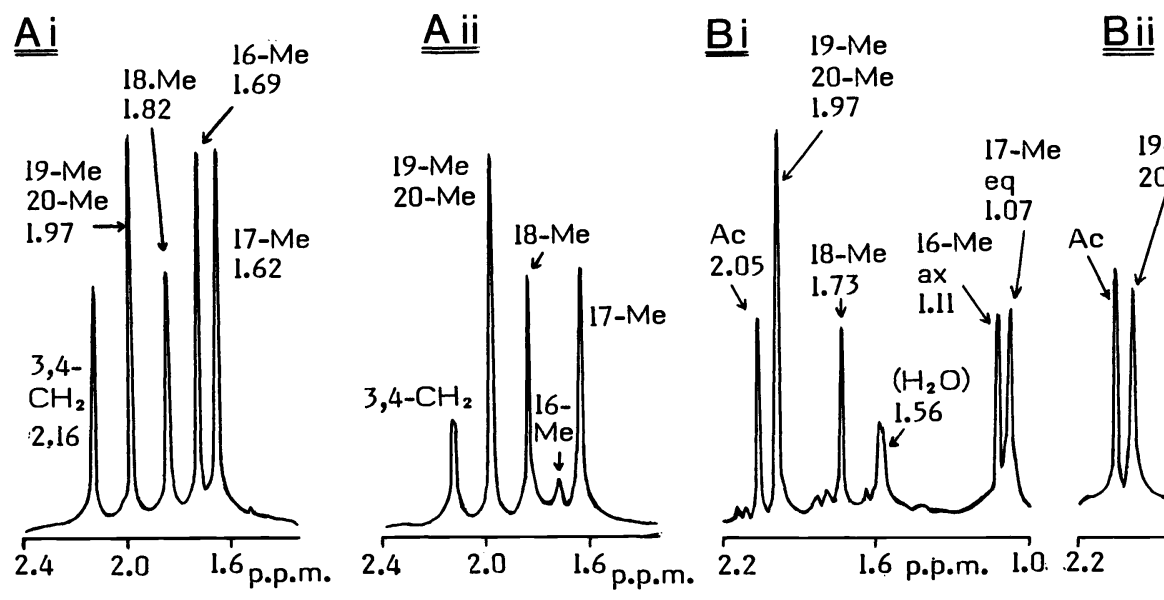

Fig.l. Methylproton region of the $\mathrm{l}_{H}$ NMR spectra of : $\underline{A}$-lycopene, $\underline{B}$-zeaxanthin : (i) normal, (ii) from Flavobacterium grown on $\left[\mathrm{l}_{\mathrm{H}}\right]$-glucose and ${ }^{2} \overline{\mathrm{H}}_{2} \mathrm{O}$.

Distinction between C-I methyl groups in zeaxanthin. This approach was then applied to the cyclic carotenoids, first zeaxanthin. This compound was isolated from Flavobacterium RISI9 grown on ${ }_{2} \mathrm{H}_{2} \mathrm{O}$ and $\left[{ }^{l_{H}}\right]$-glucose, without the addition of nicotine, and the ${ }^{1 H}$ NMR spectrum of its diacetate determined. As with lycopene, virtually no $l_{H}$ was present at the positions derived from C-5 of MVA (C$7, \mathrm{II}, 15)$. The C-I methyl substituents in zeaxanthin diacetate are not equivalent and give separate signals in the $l_{H}$ NMR spectrum (Fig IB). The spectrum of the $2 \mathrm{H}$-enriched sample clearly showed the signal at 1.075 p.p.m. due to the $\mathrm{C}-1$ equatorial methyl group (C-17) present, but that normally found at 
1.106 p.p.m. due to the C-I axial methyl group (C-16) was of very low intensity, i.e. contained very little $\mathrm{l}_{\mathrm{H}}$. This therefore identifies the equatorial C-17 as the C-1 methyl substituent derived from C-3' of MVA and the axial C-16 as the one derived from C-2 of MVA. This confirms the result previously obtained by labelling with [2-13C] MVA, and proves the validity of this novel method of determining isoprenoid labelling patterns and distinguishing between the C-I methyl substituents on the carotenoid ring.

Stereochemistry of the C-I methyl groups in decaprenoxanthin. We have now used the same method to investigate the behaviour of the C-1 methyl groups during the biosynthesis of the $\mathrm{C}_{50}$-ring carotenoid decaprenoxanthin in Flavobacterium (Cellulomonas) dehydrogenans, by growing this organism on [ $\left.{ }^{1} \mathrm{H}\right]-$ glucose and $2 \mathrm{H}_{2} \mathrm{O}$. Even though in this case the $2 \mathrm{H}:{ }^{1} \mathrm{H}$ ratio was shown by mass spectrometry to be considerably greater $(30: 42)$, the $\mathrm{l}_{\mathrm{H}}$ NMR signals due to the hydrogen substituents at $\mathrm{C}-7,11$ and 15 (derived from C-5 of MVA) were of much lower intensity than the others. In addition, the signal at 4.04 p.p.m. due to $\mathrm{CH}_{2} \mathrm{OH}$ was of much lower intensity than that of the $\mathrm{CH}_{3}$ group of the $\mathrm{C}_{5}$ sidechain at 1.68 p.p.m., confirming that these groups retain their individuality and arise via $\mathrm{C}-2$ and $\mathrm{C}-3^{\prime}$ of $\mathrm{MVA}$, respectively. The two C-l methyl substituents of decaprenoxanthin give well separated $l_{H}$ NMR signals at 0.75 and 0.95 p.p.m. The signal at 0.95 p.p.m. in the spectrum of the $2 \mathrm{H}$-enriched sample was of considerably greater intensity than that at 0.75 p.p.m. Although the assignments have not yet been proved rigorously, the signal at 0.75 p.p.m. is tentatively attributed to the C-1 axial methyl group (C-16), that at 0.95 p.p.m. to the equatorial C-17. This leads to the conclusion that the $C-1$ axial methyl group, i.e. that in the $\alpha$-position, is the one derived from $C-2$ of MVA, and the $C-1(\beta)$ equatorial methyl group arises from $C-3^{\prime}$ of MVA. If the assignments are correct this result for the $C_{50} \varepsilon-r i n g$ is in agreement with that obtained previously from the experiment involving incorporation of [2-13 C]-MVA into the $\beta$-ring $C_{50}$ carotenoid c.p. 450.

\section{Use of $\left[{ }^{13} \mathrm{C}_{6}\right]$-glucose to generate $\left.{ }^{\left[{ }^{13} \mathrm{C}\right.} 2\right]$-acetate}

The second of these strategies employs ${ }^{3} \mathrm{C}$ labelling from $\left[{ }^{13} \mathrm{C}_{6}\right]$-glucose to produce a suitably labelled endogenous substrate, in this case $\left[{ }^{13} \mathrm{C}_{2}\right]$-acetate, for conversion into carotenoid. In studies of other classes of compounds, notably polyketides but also isoprenoid fungal metabolites, extensive use has been made of incubations with acetate uniformly enriched with ${ }^{13} \mathrm{C}$ in both carbon atoms. The substrate used usually contains $10-20 \%$ of $\left[{ }^{13} \mathrm{C}_{2}\right]$-acetate molecules. When the ${ }^{13} \mathrm{C}$ NMR spectrum of the product is examined, those carbon atoms that are derived from an intact acetate unit show ${ }^{13} \mathrm{C}-{ }^{13} \mathrm{C}$ coupling and can easily be distinguished. ${ }^{13} \mathrm{C}-{ }^{13} \mathrm{C}$ coupling between carbon atoms from different acetate units is low or negligible. In the case of isoprenoids, three acetate units give rise to a molecule of MVA. One carbon atom is later lost in the formation of the 'isoprene unit'. In this $C_{5}$ unit, four of the carbon atoms are formed from two intact acetate units. The fifth, that equivalent to $C-2$ of MVA, is not and therefore carbon atoms derived from this position, C-2 of MVA, can be distinguished by the lack of C-C coupling in the ${ }^{13} \mathrm{C}$ NMR spectrum of the product. This approach has not yet been used in the carotenoid field because of the inefficient incorporation of acetate by most carotenogenic organisms. However, we have recently achieved similar results by culturing microorganisms on generally labelled $\left.{ }^{1{ }^{13}} \mathrm{C}_{6}\right]-g$ lucose as carbon source. The biosynthesis of carotenoids can then be summarized as

$$
\text { glucose } \longrightarrow \text { acetate } \longrightarrow \text { MVA carotenoid }
$$

Provided acetate is formed direct from the glucose as an intact $C_{2}$ unit to an appreciable extent, the system can be considered to be using endogenous $\left[{ }^{13} \mathrm{C}_{2}\right]$-acetate as substrate and results obtained should be equivalent to those that would be obtained if exogenous $\left[{ }^{13} \mathrm{C}_{2}\right]$-acetate were supplied and utilized. Some metabolic conversion of glucose to acetate via $C_{1}$ units, either by direct breakdown or via cycles, is inevitable, and will give the equivalent of $\left[{ }^{13} \mathrm{C}_{1}\right]$-acetate units, but so long as an appreciable proportion of the acetate formed and used in carotenoid synthesis arises directly as $C_{2}$ units then, in any product, those carbon atoms derived via C-2 of MVA will be distinguishable by the lack of, or low level of $C-C$ coupling in the ${ }^{13} \mathrm{C}$ NMR spectrum, compared with carbon atoms derived from the intact $C_{2}$ units that make up the rest of the MVA molecule. A somewhat similar procedure has been used to demonstrate that the simple terpenoid pentalenolactone is biosynthesized via mevalonate. (Ref. 26).

Labelling pattern in zeaxanthin. Fig $2 \mathrm{~A}$ illustrates the results of an experiment in which Flavobacterium R1560 was grown on a substrate consisting of $\left[{ }^{13} \mathrm{C}_{6}\right]$-glucose (20\%) and natural abundance $\left.{ }^{12} \mathrm{C}_{6}\right]$-glucose $(80 \%)$, and the main carotenoid produced, zeaxanthin, isolated. Under these conditions, the maximum possible extent of coupling between adjacent carbon atoms arising from different $C_{2}$ units or single carbon atoms (C-2 of MVA) is $4 \%$, whereas those carbon atoms arising from an intact $\mathrm{C}_{2}$ unit should show extensive $\mathrm{C}-\mathrm{C}$ coupling. In the ${ }^{13} \mathrm{C}$ NMR spectrum of this zeaxanthin, most of the signals are seen as apparent triplets, consisting of a central signal for the uncoupled carbon flanked by satellite peaks due to C-C coupling. In some cases, however, the satellite peaks are virtually absent, showing the absence of C-C coupling. The signals showing no coupling are, as predicted, those of carbons derived from C-2 of MVA. In particular, the C-1 methyl substituents can again be distinguished, the signal at 28.72 p.p.m. due to the $1 \alpha$, axial, substituent (C-16) showing virtually no $C-C$ coupling, whereas that at 30.25 p.p.m. due to $C-17$, the $1 \beta$, equatorial substituent, shows substantial coupling. This therefore confirms the labelling pattern obtained by using [2-13 C]-MVA as substrate, and establishes the validity of the arguments and procedure for the use of $\left[{ }^{13} C_{6}\right]$-glucose as substrate. 


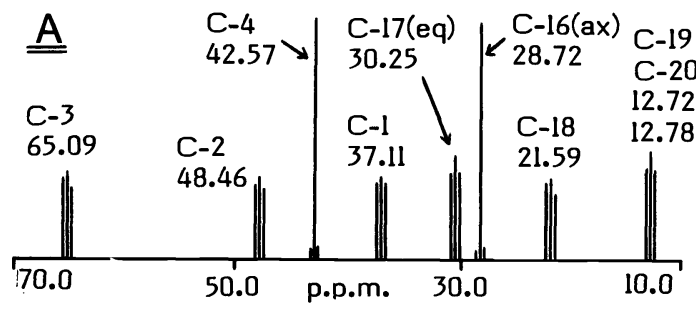

C-8

138.51
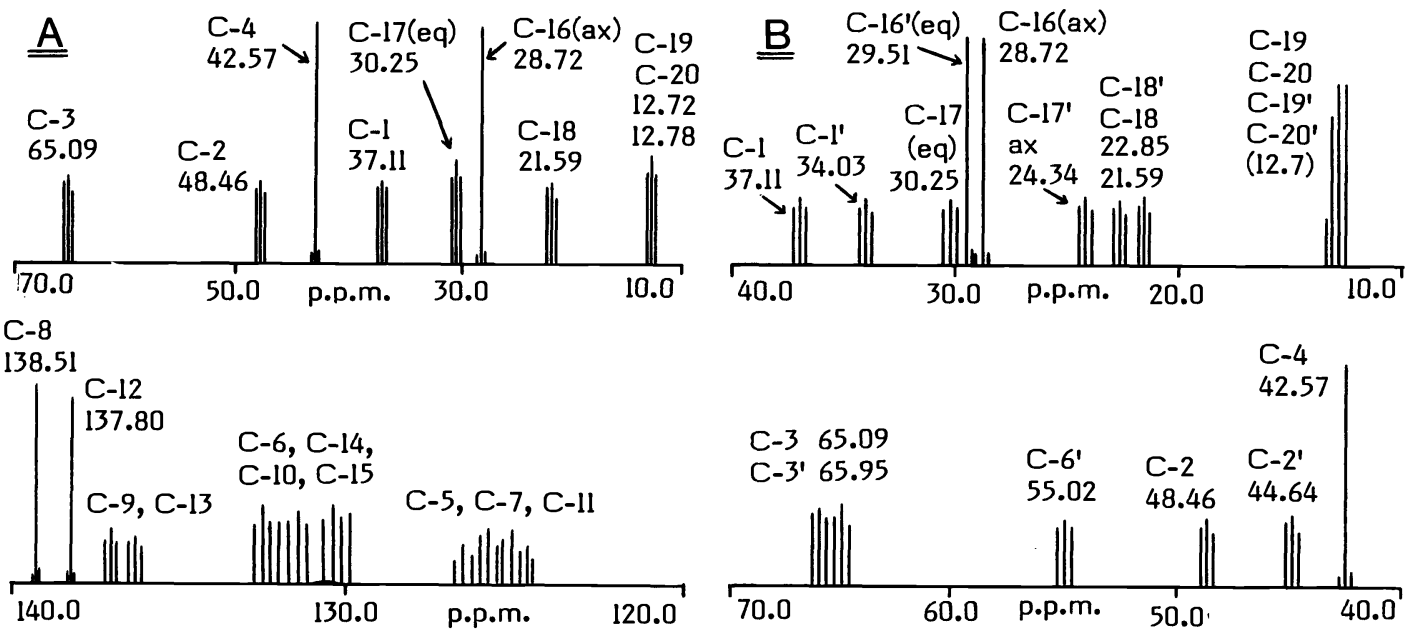
Fig.2. Partial ${ }^{13} \mathrm{C}$ NMR spectra of : A-zeaxanthin from Flavobacterium, B-lutein from
Scenedesmus obliquus, grown on $\left.{ }^{13} \mathrm{C}_{6}\right]$-glucose.

Stereochemistry of the C-I methyl groups in lutein. This procedure has now been used to tackle one of the outstanding problems in the stereochemistry of carotenoid biosynthesis, the behaviour of the $C-1$ methyl groups during formation of the $\varepsilon$-ring. No system has yet been found that will incorporate exogenous MVA or acetate efficiently enough to allow standard ${ }^{13} \mathrm{C}$ enrichment experiments. In particular Scenedesmus obliquus, which we have used to establish the stereochemistry of introduction of hydrogen at C-2' during cyclization to form the $\varepsilon$-ring, will not incorporate MVA or acetate. Although when grown in the light it uses only $\mathrm{CO}_{2}$ as carbon source, it will grow in the dark on glucose as substrate and still produce the normal range of chloroplast cyclic carotenoids, including lutein. The alga (wild type) was therefore grown on medium containing $\left[{ }^{13} \mathrm{C}_{6}\right]-g l u c o s e(20 \%)$ and $\left[{ }^{12} \mathrm{C}_{6}\right]$-glucose $(80 \%)$ as carbon source, and the lutein was isolated and purified and its $13 \mathrm{C}$ NMR spectrum determined ( $F$ ig 2B). The signals due to carbon atoms derived from C-2 of MVA could clearly be distinguished by the absence of significant C-C coupling satellites. This confirms the isoprenoid labelling pattern and the origin of $C$ $4,8,12,4^{\prime}, 8^{\prime}$ and $12^{\prime}$ of the lutein from C-2 of MVA. The four C-1 methyl substituents, from the $\beta$. and $\varepsilon$-rings, could also be distinguished. As with zeaxanthin from Flavobacterium, it was C-16 (the C-1 axial methyl group) of the 3-hydroxy- $\beta$-ring (28.72 p.p.m.) that showed virtually no $C-C$ coupling and hence arises from C-2 of MVA. Of the two C-1 methyl substituents of the $\varepsilon$-ring the signal (29.51 p.p.m.) of C16 ', the l' $\alpha$-methyl substituent (in this case equatorial) was again distinguished by the lack of $C-C$ coupling, and is therefore the one arising from C-2 of MVA and C-16 (trans to the main carbon chain) of the acyclic precursor. The C-17' (I' $\beta$ - methyl group) signal (24.34 p.p.m.) did show extensive coupling. Thus, even though the incorporation of exogenous substrate was not possible, this procedure has allowed us to prove that the stereochemical behaviour of the $C-1$ methyl groups during cyclization is the same in the $\beta$ - and $\varepsilon$-rings.

\section{CONCLUSIONS}

A short article such as this can present only a brief outline of published work on the use of stable isotopes in carotenoid biochemistry, and of unpublished work from the author's laboratory. It should, however, show how powerful these techniques are in giving a direct and unequivocal answer to many of the questions posed. The two-phase $2 \mathrm{H}_{2} \mathrm{O}$ labelling procedure has already proved useful in demonstrating carotenoid transformations during biosynthesis and during developmental processes such as chloroplast maturation. Much work remains to be done in the latter area. Perhaps the main achievement of the stable isotope work so far has been the elucidation of the stereochemistry of cyclization to form both $\beta$ - and $\varepsilon$-rings in the $C_{40}$ and $C_{50}$ carotenoid series. The application of the range of procedures that we have developed to investigate isoprenoid labelling patterns and details of the stereochemistry of biosynthesis of other carotenoids is to be anticipated. The results obtained to date are summarized in Fig. 3. No difference was found between the $\beta$ and $\varepsilon$-rings of the $C_{40}$ series in the stereochemistry of hydrogen substitution at C-2 or in the behaviour and disposition of the C-1 methyl groups. The suggestion that these different ring types are formed by alternative proton loss from C- 6 or C- 4 of the same 'carbonium ion' intermediate therefore remains valid, and the stereochemistry of folding of the acyclic precursor to produce this intermediate conforms to the $C_{2}(E, E) / B_{2}(E, Z)$ alternative in the series of possibilities tabulated by Eugster (Refs. 10, II) as an extension of those we proposed some years ago (Ref. 27). Perhaps the most interesting and surprising finding was that the behaviour of the $C-1$ methyl substituents during formation of the $\beta$ - and $\varepsilon$-rings in the $C_{50}$ carotenoids, 'c.p. 450 ' and decaprenoxanthin, appears to be the same as for the $C_{40}$ series even though the stereochemistry of substitution at $\mathrm{C}-2$ is opposite. If the tentative NMR assignments are confirmed, these $\mathrm{C}_{50}$ molecules 


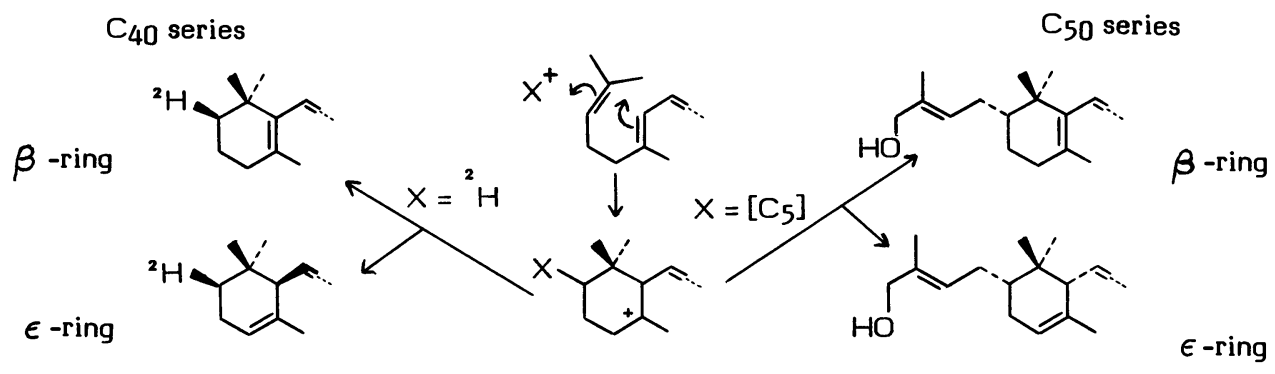

Fig.3. Comparison of the stereochemistry of cyclization to form $\beta$ - and $\varepsilon$-rings in $C_{40}$ and $C_{50}$ carotenoids.

therefore do not conform to any of the possible patterns listed by Eugster. Other features therefore need to be considered when attempting to define the stereochemical course of cyclization in the $C_{50}$ carotenoid series. Any attempt at explanation at this stage would be purely speculative and premature; further experimentation is obviously needed.

\section{ACKNOWLEDGEMENTS.}

I am most grateful to the coworkers responsible for most of the experimental work outlined in this article:- Drs. Bill Lockley, Narendra Patel, Ann Mundy and Susan Pollard, and Mr. Michael Browne, -also to our colleagues Gary Harriman, Malcolm Rose and Mark Prescott for determination of mass spectra, to Dr. Gerhard Englert (Basel) and Dr. Peter Bevan (Southampton) for determination of NMR spectra, and to Dr. Englert for much patient work on the rigorous assignment of the spectra. Financial support from S.E.R.C. is acknowledged with thanks. I also wish to take this opportunity to express my gratitude to Emeritus Professor T.W. Goodwin, C.B.E., F.R.S., who first introduced me to the world of carotenoids and from whom I have learned so much.

\section{REFERENCES}

1. J. Lugtenburg, Pure Appl. Chem. this volume.

2. E.A. Shneour, Biochim. Biophys, Acta, 65, 510 (1962).

3. H.Y. Yamamoto, C.O. Chichester and T.O.M. Nakayama, Arch. Biochem. Biophys., 96, 645-9 (1962).

4. H.Y. Yamamoto, C.O. Chichester and T.O.M. Nakayama, Photochem. Photobiol., 1,53 (1962).

5. E.A. Shneour and M. Calvin, Nature, 196, 439 (1962).

6. D.I. Sapozhnikov, Dokl. Akad. Nauk. S.S.S.R., 154, 974 (1964).

7. H.Y. Yamamoto and C.O. Chichester, Biochim. Biophys. Acta, 109, 303-5 (1965).

8. K.L. Simpson, T.O.M. Nakayama and C.O. Chichester, Biochem. J., 92, 508-510 (1964).

9. G. Englert, in Carotenoid Chemistry and Biochemistry (Eds. G. Britton and T.W. Goodwin) pp. 107134, Pergamon, Oxford (1982).

10. C.H. Eugster, Pure Appl. Chem. 51, 463-506 (1979).

11. C.H. Eugster, in Carotenoid Chemistry and Biochemistry (Eds. G. Britton and T.W. Goodwin), pp. 126, Pergamon, Oxford (1982).

12. G. Britton, T.W. Goodwin, W.J.S. Lockley, A.P. Mundy, N.J. Patel and G. Englert, J. Chem. Soc. Chem. Commun., 27-28 (1979).

13. E.C. Grob and R. Butler, Helv. Chim. Acta 38, 1313 (1955); 39, 1975 (1956).

14. A.G. Andrewes, S. Liaaen-Jensen and G. Borch, Acta Chem. Scand., B28, 737-742 (1974).

15. A.G. Andrewes, S. Liaaen-Jensen and O.B. Weeks, Acta Chem. Scand., B29, 884-6 (1975).

16. S. Hertzberg and S. Liaaen-Jensen, Acta Chem. Scand., B31, 215-8 (1977).

17. J.J. Katz and H.L. Crespi, Science 151, 1187-1194 (1966).

18. W.A. Svec, A.L. Harkness, H.H. Strain and J.J. Katz, Org. Mass Spectrom., 6, 843-851 (1972).

19. G. Britton, W.J.S. Lockley, N.J. Patel and T.W. Goodwin, FEBS Letters 79, 281-3 (1977).

20. N.J. Patel, G. Britton and T.W. Goodwin, Biochim. Biophys. Acta 760, 92-96 (1983).

21. G. Britton, W.J.S. Lockley, N.J. Patel, T.W. Goodwin and G. Englert, J. Chem. Soc. Chem. Commun., 655-6 (1977).

22. G. Britton, R. Powls and R.M. Schulze, Arch. Microbiol., 113, 281-4 (1977).

23. G. Britton and A.P. Mundy, Dev. Plant Biol., 6, 345-350 (1980).

24 G. Britton, W.J.S. Lockley, R. Powls, T.W. Goodwin and L.M. Heyes, Nature, 268, 81-2 (1977).

25. G. Britton in Photosynthesis (ed. G. Akoyunoglou) $\underline{5}, 261-6$ (1981).

26. D.E. Cane, T. Rossi and J.P. Pachlatko, Tetrahedron Letters, 3639-3642 (1979).

27. G. Britton in Aspects of Terpenoid Chemistry and Biochemistry, (ed. T.W. Goodwin) pp. 254-289. Academic Press, London (1971). 